\title{
An evaluation of sleep disturbance on in-patient psychiatric units in the UK
}

\author{
Sam Horne, ${ }^{1}$ Katherine Hay, ${ }^{2}$ Stuart Watson, ${ }^{1,2}$ Kirstie N. Anderson ${ }^{3}$
}

BJPsych Bulletin (2018) 42, 193-197, doi:10.1192/bjb.2018.42

${ }^{1}$ Newcastle University, UK;

${ }^{2}$ Northumberland Tyne and Wear NHS Foundation Trust, UK; ${ }^{3}$ Newcastle upon Tyne Hospitals NHS Foundation Trust, UK

Correspondence to Dr Kirstie N. Anderson (kirstie.anderson@nuth. nhs.uk)

First received 15 Jan 2018, final revision 14 Mar 2018, accepted 11 May 2018

(c) The Authors 2018. This is an Open Access article, distributed under the terms of the Creative Commons

Attribution-NonCommercial-

NoDerivatives licence (http://

creativecommons.org/licenses/by-ncnd/4.0/), which permits noncommercial re-use, distribution, and reproduction in any medium, provided the original work is unaltered and is properly cited. The written permission of Cambridge University Press must be obtained for commercial re-use or in order to create a derivative work.
Aims and method Sleep disturbance is common on in-patient psychiatry wards. This study explored subjective and objective patterns of sleep disturbance and contributory environmental factors. Participants were recruited from mental health acute admission wards and had a range of subjective and objective assessments of sleep. Light intensity and noise levels were measured to characterise potential environmental causes for poor sleep.

Results We recruited 20 patients; 15\% were high risk for obstructive sleep apnoea. Nineteen participants reported poor sleep quality on the Pittsburgh Sleep Quality Index, and 90\% had significant sleep fragmentation with objective measures. Inside light levels were low (day $<200$ lux and night $<10$ lux). Night sound levels were 40-90 db.

Clinical implications Sleep disturbance was highly prevalent. Increased awareness of sleep disorders is needed. Modifiable environmental factors on the ward were implicated, therefore increased awareness and a change of approach to sleep disturbance in in-patient psychiatry is recommended.

\section{Declaration of interest None.}

Keywords Sleep disturbance; accelerometry; in-patient psychiatry.

\section{Sleep disturbance in psychiatric disease}

The normal timing and duration of sleep is vital for normal mental health. ${ }^{1}$ Poor sleep is a core symptom of many psychiatric conditions. However, sleep disorders such as insomnia, obstructive sleep apnoea, REM sleep behaviour disorder and circadian rhythm disorder are also common and remain underdiagnosed. ${ }^{2}$ The frequent comorbidity of disrupted sleep and psychiatric disorder, ${ }^{3}$ and the negative prognostic effect of disrupted sleep, including on suicide risk, ${ }^{4,5}$ all demonstrate the challenge and importance for mental health professionals of assessing and protecting sleep when patients require admission.

Admission to a psychiatric unit represents both a particular need and opportunity to identify and manage disturbed sleep, including sleep disorders. However, it has been noted that sleep enhancement does not appear to be a priority on acute admission wards. In many trusts, for instance, hourly overnight monitoring of patients remains routine and is paradoxically used because of the risk of harm to the patient.

\section{Overnight monitoring on psychiatry wards}

Concerns about the risk of suicide ${ }^{6}$ place a perceived burden on staff in in-patient units to regularly monitor patients overnight. This monitoring can in itself further disrupt sleep and may therefore affect recovery. The standards for the safe care of patients on psychiatric wards by the Royal College of Psychiatrists ${ }^{7}$ do not include or specify protected sleep time, and current National Institute of Health and Care Excellence recommended in-patient pathways ${ }^{8}$ in acute hospitals make no mention of sleep beyond a single survey question about night-time disruption by staff. The best way to monitor, measure and enhance sleep on in-patient wards is unclear. We aimed to objectively assess sleep and the ward environment, and attempted to characterise patterns and causes of sleep disturbance on in-patient psychiatric wards.

\section{Method}

Participants were sequentially recruited from four acute psychiatric wards, two male and two female, in two separate in-patient units within Newcastle, Tyne and Wear NHS Foundation Trust. Recruitment was opportunistic, with all patients meeting the inclusion criteria being considered for the study. Inclusion criteria required that participants were aged 18-70 years, able to provide written informed consent and were fluent in English. All participants had capacity to consent and written consent was obtained after a period of at least 24 hours to consider the details of the study. 
Patients were approached after $72 \mathrm{~h}$ on the ward and were included in the study if they were expected to be staying on the ward for at least a 1-week period.

Sleep quality was then assessed with the Pittsburgh Sleep Quality Index (PSQI) ${ }^{9}$ and the risk of sleep apnoea was assessed with the STOP-BANG questionnaire. ${ }^{10}$ Using the PSQI, participants were categorised as having normal sleep (score $<5$ ), some level of sleep disturbance (score $\leq 10)$ or severe sleep disturbance (score >10). The STOP-BANG questionnaire is a validated screening tool for sleep apnoea. Participants scoring $\geq 5$ were considered high risk for obstructive sleep apnoea. If a participant was found to be high risk for obstructive sleep apnoea, this was communicated by letter to their general practitioner.

Objective sleep quality was assessed with a wrist accelerometer with a light monitor (GENEactiv original). Participants were asked to wear the watch continuously for a week. Participants who continuously wore the wrist accelerometer for five or more continuous days and nights were included in the study (20 out of 32 ). The algorithm for analysis has been published and used to assess sleep-wake disturbance in those with and without mental health problems. $^{11,12}$ Total sleep time, daytime naps and sleep fragmentation were all measured. Twelve participants were excluded because of incomplete data: patients were discharged sooner than expected, and for two participants, wearing the watch itself caused agitation and was not tolerated.

Participant medical records were accessed for basic demographics, current and past psychiatric history and any reference to sleep difficulties.

Sound level and light level were identified as potential environmental factors that could affect sleep quality. Monitoring of sound level was carried out with a decibelmetre (Extech 407760). The night-time noise level between 21.00 and 09.00 was analysed over three consecutive nights on two separate wards. Data was measured continuously to allow both baseline average to be calculated and spikes of higher decibel levels to be seen. An unused bedroom was used to avoid disturbing patients with the monitoring equipment itself.

Daytime and night-time light levels were measured with a portable lux meter (MINOLTA chroma meter cs-100). For daytime measurements, the lux level within the bedrooms, communal areas and dining areas across all the wards was measured. Once selected, the same areas were used for repeated measurements. Measurements were averaged over two separate days to account for any differences in inside light level attributable to outside light level. Light measurements were also taken at night-time after 22.00. Night-time measurements focused entirely on the patient bedrooms and were taken on two separate occasions on each ward.

Ethical approval was granted for the study by the East of England - Cambridge East Research Ethics Committee (approval number IRAS 222122).

\section{Results}

Over the study period (April 2017-July 2017), there were 150 admissions to the four study wards. Of these, only 40 met the inclusion criteria, mostly because of lack of capacity to consent or short duration of stay. Thirty-two patients agreed to participate and completed both the PSQI and STOP-BANG questionnaire. Twenty participants then provided five or more continuous days of accelerometry recording, and this group were analysed in more detail and the results are presented below. The median age of participants was 49 years (range 30-70 years). Female participants outnumbered male participants by a factor of just over 2:1, (13 women and 7 men). Depression was the most common primary diagnosis, followed by bipolar affective disorder and schizophrenia. Only a single patient had a diagnosis of mania during the admission.

All participants completed the STOP-BANG questionnaire, and three (15\%) scored $\geq 5$ and were therefore at high risk for having obstructive sleep apnoea. All three were male, aged 48, 51 and 57 years, respectively, and all had objectively abnormal sleep as measured by accelerometry, with prolonged sleep period and fragmented night sleep.

Nineteen participants had a global PSQI score $\geq 5$, representing poor sleep quality, and 12 scored $>10$ in the more severe range. The single patient with mania self-rated sleep as normal, with a PSQI score of 4 , but had objectively abnormal sleep on actigraphy, with irregular, short sleep periods. The range of PSQI scores was between 4 and 18 . Fourteen participants scored $\geq 10$ (severe sleep disturbance). The separate domains of the PSQI were also assessed with previously published subscales looking at sleep efficiency, daily disturbances and perceived sleep quality to see if there was one domain more likely to cause sleep disturbance. ${ }^{13}$ Patients all had multiple causes of sleep disturbance including, but not exclusive to, the ward environment. All participants were prescribed some form of psychotropic medication, with 18 participants receiving multiple medications and 8 participants prescribed zopiclone for sleep disturbance.

\section{Actigraphy}

Twenty out of 32 participants wore the accelerometers continuously for five or more days. Representative actograms from six participants are shown in Fig. 1. Variable patterns of sleep and physical activity were easily visualised over 57 days of accelerometry. Participants were categorised as long (average duration of $>10 \mathrm{~h}$ sleep in a $24 \mathrm{~h}$ period), short ( $<6 \mathrm{~h}$ within a $24 \mathrm{~h}$ period) or normal sleepers, with regards to the total amount of sleep achieved in $24 \mathrm{~h}$. Eighteen participants had fragmented sleep during their main sleep period, with frequent night wakening. No patient had an average sleep period of less than $6 \mathrm{~h}$ when on the ward, although three self-rated their sleep as short. Three were (15\%) long sleepers and seven (35\%) had sleep duration between 6 and $10 \mathrm{~h}$.

Actigraphy results showed marked sleep fragmentation and night-time awakenings. It was apparent that light levels were intermittently increased during the night within the patient rooms, consistent with a disturbed night and often coinciding with night-time observations. Many of the long sleepers had fragmented nights with frequent awakenings. Prolonged daytime naps were also seen. 


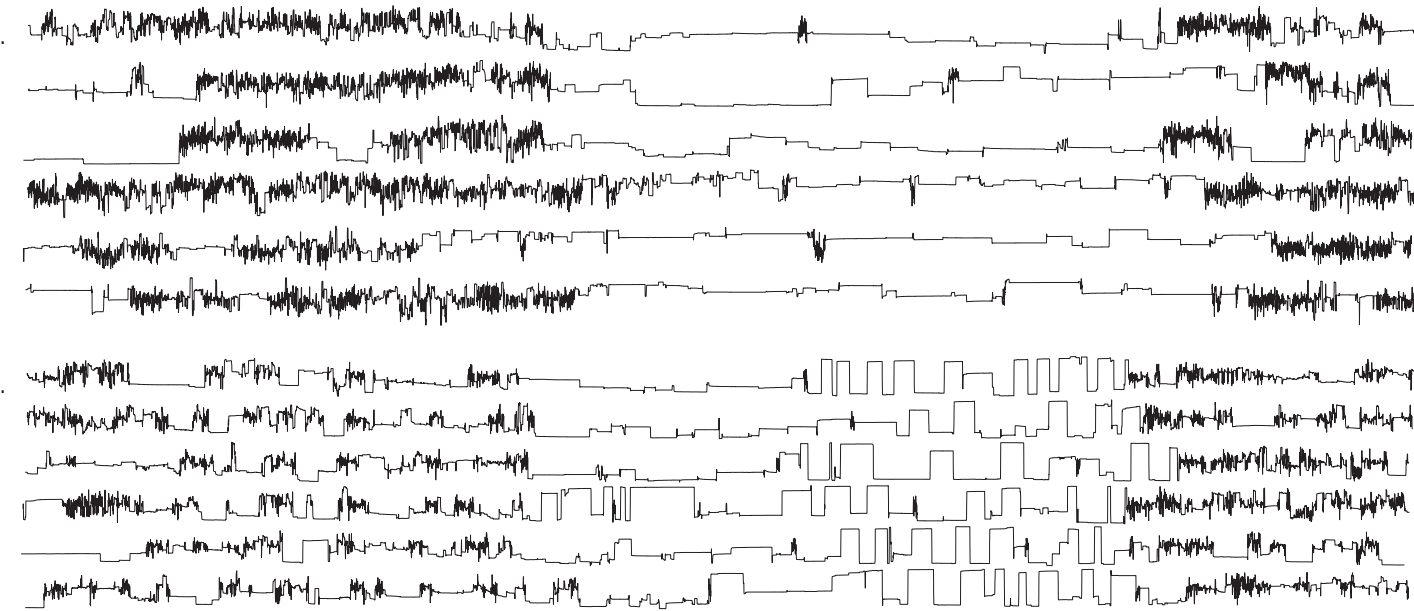

C.

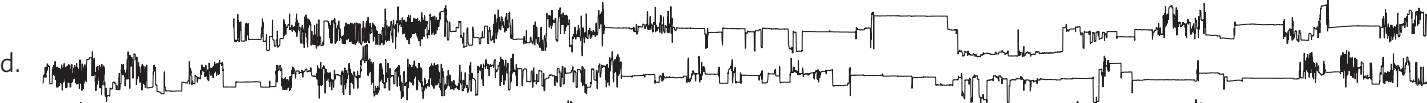

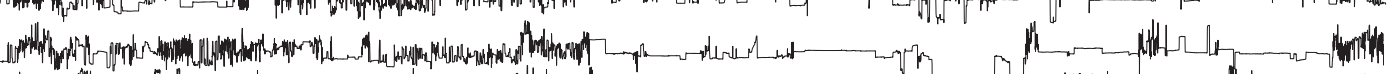

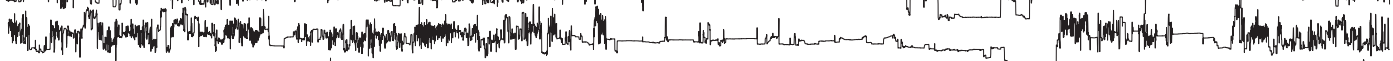

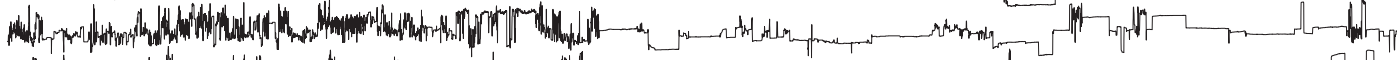

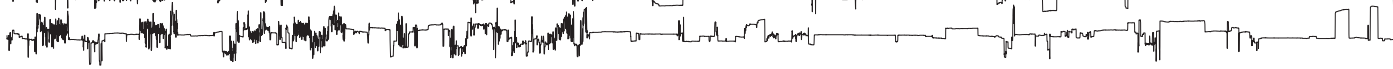

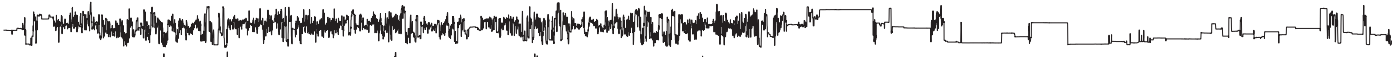

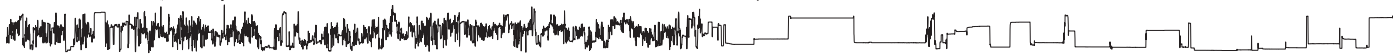

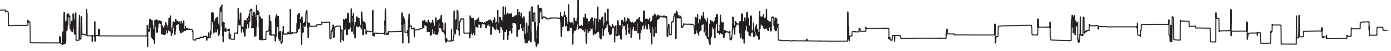
A

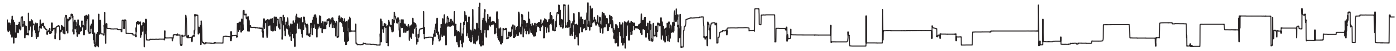
乙

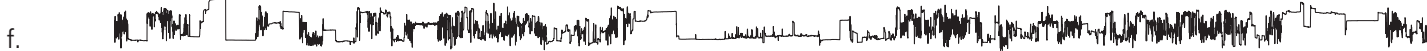

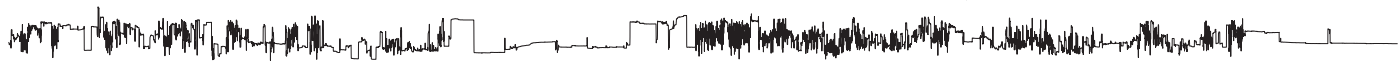

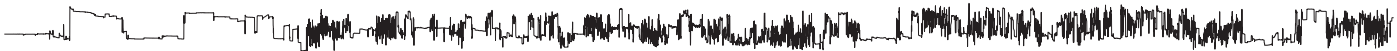

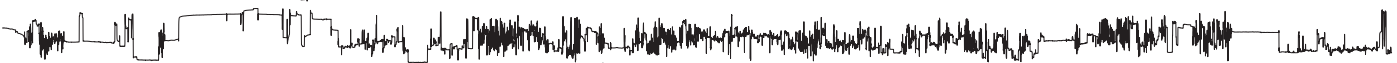

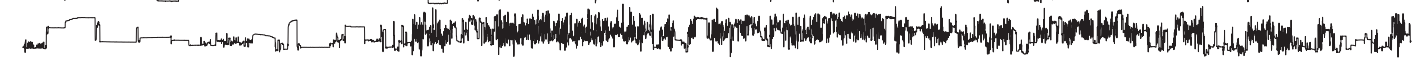

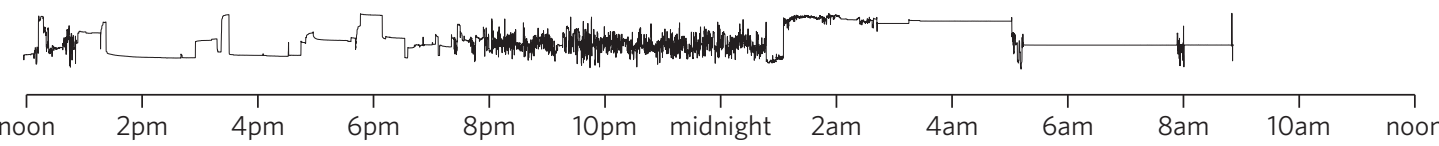

Fig. 1 Accelerometry data from six patients showing variable sleep-wake patterns. For all participants, wrist accelerometry data is shown plotted over sequential days from 12.00 to 12.00 over a $48 \mathrm{~h}$ period to show the overnight sleep period. Periods of sleep can clearly be seen, as well as sleep fragmentation and night-time awakenings. (a) Female, 70 years of age with a diagnosis of schizophrenia. A pattern of prolonged sleep with occasional night-time awakenings is seen and daytime naps. (b) Female, 59 years of age with depression. There is reduced daytime activity with naps, and also a very fragmented and prolonged total sleep time. (c) Male, 44 years of age with schizophrenia. There is a shorter sleep time, higher levels of daytime activity and a less fragmented sleep period. (d) Male, 53 years of age with depression. There is a pattern of prolonged sleep, fragmented night-time sleep and prolonged daytime naps. (e) Male, 39 years of age with schizoaffective disorder. There is a more delayed sleep onset between 00.00 and 02.00 , with a wake time at 12.00. ( $f$ ) Male, 48 years of age with depression, alcohol dependence and post-traumatic stress disorder. A striking pattern of delayed sleep phase is seen, with the sleep period during the day and high levels of activity at night. 


\section{Light and noise levels across the wards}

The sound level data showed that the wards maintained an overnight decibel level within a range of $38-70 \mathrm{db}$, between 21.00 and 05.00, with occasional spikes that reached $90 \mathrm{db}$. There was variation across the wards and a gradual rise in noise level between 03.00 and 07.00 across the wards to $45 \mathrm{db}$ as an average background. Some causes of the brief spikes in noise included equipment alarms and safety doors closing.

Light intensity was measured across the entire unit and the data is summarised in Table 1. Throughout the in-patient areas, there was a very low level of light intensity across all communal areas. None of the indoor areas had a light intensity similar to that outdoors, however the light levels within the bedroom were very low.

\section{Discussion}

Subjective assessment of sleep quality showed disturbed sleep for almost all participants (19 out of 20), with marked sleep disturbance reported by $60 \%$. The PSQI was tolerated well and completed by all patients, suggesting a variety of factors affecting sleep quality. Objective measures of sleep also showed significant sleep disturbance, and in particular sleep fragmentation, when measured with accelerometry. One potential cause was the hourly nightly observations, as fragmentation appeared regular and consistent in many participants along with brief periods of increased light levels, and all participants were subject to these hourly checks. We accept that this is simply one potential cause of sleep disturbance, as that it was not possible to correlate actigraphy at all times with the light and noise measurements, given the prolonged period of actigraphy over a 4-month period. However, if patient sleep is being disturbed by these checks, the implication is that this process could be

\begin{tabular}{|lcccc|}
\hline Table 1 & Mean light levels in different locations \\
Location & \multicolumn{5}{c|}{ Mean light levels (lux) } \\
\cline { 2 - 6 } & Ward A & Ward B & Ward C & Ward D \\
\hline $\begin{array}{l}\text { Daytime (approximate } \\
\text { time) }\end{array}$ & 11.15 & 15.35 & 15.00 & 11.15 \\
\hline $\begin{array}{l}\text { Bedroom - door closed } \\
236\end{array}$ & 7 & 11 & 38 \\
\hline Bedroom - door open & 228 & 8 & 10 & 44 \\
\hline Bedroom - light on & 231 & 46 & 47 & 52 \\
\hline Communal area & 373 & 26 & 164 & 221 \\
\hline Dining room & 74 & 50 & 120 & 58 \\
\hline Outside & 11528 & & & \\
\hline $\begin{array}{l}\text { Night-time (approximate } \\
\text { time) }\end{array}$ & 23.15 & 22.40 & 22.40 & 23.30 \\
\hline Bedroom - door closed & 0 & 0 & 0 & 0 \\
\hline Bedroom - door open & 0.3 & 0.1 & 0.8 & 0 \\
\hline Bedroom - side light on & 3.7 & 3.6 & 5 & 4.1 \\
\hline
\end{tabular}

On each ward, measures were taken at approximately the same time on one cloudy day and one sunny day and the mean was calculated (May 2017). At night-time, measures were also taken on two separate days and a mean calculated. counterproductive, slowing recovery because of the effect of sleep disturbance on mental health. The importance of regular observations is acknowledged in those at high risk of harm (accounting for a number of patients on the study wards); however, the most recent review from the National Confidential Enquiry into Suicide ${ }^{6}$ emphasised the need for a personalised care plan and the avoidance of routine checklists. Reports from this group have suggested that routine observations are not helpful and yet they remain widespread across acute mental health trusts throughout the UK.

There are clearly a number of potential reasons for sleep disturbance within an in-patient psychiatric population, including the mental health symptoms themselves and the medication used to treat symptoms; however, primary sleep disorders such as obstructive sleep apnoea are common in those with severe and enduring mental health problems, and will affect sleep quality and contribute to nocturnal hypertension. ${ }^{14,15}$ Risk factors include male gender, body mass index and obesity and age $>50$ years. It is notable that $15 \%$ of patients were high risk for sleep apnoea based on sleep questionnaire screening and all were male. Any in-patient admission offers an opportunity for physical health assessment, with increasing recognition of the poor cardiometabolic health of many with psychiatric disease. ${ }^{16,17}$ Obstructive sleep apnoea may be ameliorated by rationalising medication that may contribute to obesity and snoring (e.g. benzodiazepines) and via the effective, evidenced-based therapy of continuous positive airways pressure.

The ward environment may also be a modifiable part of sleep disturbance. It seems likely that sound levels were a contributory factor to sleep fragmentation. The sound level for both wards exceeded the recommended night-time noise level for a hospital ward of $30 \mathrm{db}^{18}$ They remained between $40 \mathrm{db}$ (equivalent to the sound in a library) and $70 \mathrm{db}$ (equivalent to the noise produced by a vacuum cleaner) during the overnight period. The occasional spikes of a much louder noise, which reached up to $90 \mathrm{db}$ (equivalent to the noise produced by a food blender or a lawn mower), are likely to disrupt sleep. There have been previous reports highlighting concerns about noise pollution affecting health in hospitals. This seems an important and potentially modifiable part of night-time sleep disruption. ${ }^{19}$

The light levels recorded at night with the bedroom door shut did not exceed 1 lux. This implies that the night-time light environment of patient bedrooms is conducive for sleep when the doors are not opened for observations. At night, with the side light, the light levels recorded were $<10$ lux in the bedrooms. Daytime light levels, however, were also low, never exceeding 200 lux across the unit and $<50$ lux in the bedrooms. The retinohypothalamic tract controls circadian rhythm and is dependent on light intensity for normal function. ${ }^{20}$ Lack of exposure to adequate light intensities may contribute to the dysregulation of circadian rhythms, which is increasingly recognised in psychiatric disorders and may, in turn, contribute to the tendency toward daytime napping and irregular sleep patterns, and may affect the ability of the patient to engage with psychological or occupational therapies. ${ }^{21}$ These data suggest that the light environment of psychiatric wards should be improved and highlight the importance of time off of the ward in outside spaces with natural light. 


\section{Limitations of the study}

It was not possible to time-lock sound measurement to accelerometry data, as two different methods of recording were used. For future studies, more detailed in-patient assessments, such as video polysomnography, could be used or time-locked continuous video monitoring; however, this detail of recording may in itself cause patient agitation if symptoms such as paranoia and delusional ideation are present. Accelerometry was reasonably well tolerated but still only worn continuously for 20 out of 32 participants. Therefore, non-invasive methods of recording sleep-wake patterns are needed for this population to avoid distress. There has been only a single, smaller study that compared nursing observations, patient sleep diaries and 3 days of wrist actigraphy in eight patients in their initial 3 days on a psychiatry ward. This showed that nursing staff tended to overestimate sleep and that patient sleep improved from the first to the third night. ${ }^{22}$ This was one reason that we assessed all patients at least $72 \mathrm{~h}$ after admission.

The sample size was relatively small, and the study was not powered to look for significant differences across, for example, different diseases or those on or off of different psychotropic medication. A lack of capacity to give informed consent and short stay duration was a reason for many on an acute ward being unable to participate in the study. Therefore future studies may need to recruit over a longer time period to increase the sample size. This remains the first UK study to date to study sleep within acute psychiatry wards.

In summary, the results of this small study demonstrate high levels of sleep disturbance on in-patient psychiatric wards and variable patterns of sleep-wake disturbance. Sleep disturbance affects mental health and so should be a factor when developing care pathways; however, to date, there has been very little qualitative or quantitative research in this area. Some level of sleep disturbance may relate to ward environment and some may relate to an undiagnosed primary sleep disorder. Therefore, further studies that evaluate a sleep care plan as part of standard in-patient care are recommended.

\section{About the authors}

Sam Horne, Medical Student, Institute of Neuroscience, Newcastle University, UK; Katherine Hay, Speciality Trainee Psychiatrist, Northumberland Tyne and Wear NHS Foundation Trust, UK; Stuart Watson, Consultant Psychiatrist, Institute of Neuroscience, Newcastle University, UK and Northumberland Tyne and Wear NHS Foundation Trust, UK; Kirstie N. Anderson, Consultant Neurologist and Sleep Specialist, Stuart Watson Consultant Psychiatrist, Katherine Speciality Trainee Psychiatry, Sam Horne Medical Student, The Regional Sleep Service, Newcastle upon Tyne Hospitals NHS Foundation Trust, UK

\section{References}

1 Wulff K, Gatti S, Wettstein JG, Foster RG. Sleep and circadian rhythm disruption in psychiatric and neurodegenerative disease. Nat Rev Neurosci 2010; 11(8): 589-99.
2 Stores G. Clinical diagnosis and misdiagnosis of sleep disorders J Neurol Neurosurg Psychiatry 2007; 78(12): 1293-7.

3 Benca RM, Obermeyer WH, Thisted RA, Gillin JC. Sleep and psychiatric disorders. A meta-analysis. Arch Gen Psychiatry 1992; 49(8): 651-68; discussion 69-70.

4 Pigeon WR, Pinquart M, Conner K. Meta-analysis of sleep disturbance and suicidal thoughts and behaviors. J Clin Psychiatry 2012; 73(9): e1160-7.

5 Bernert RA, Kim JS, Iwata NG, Perlis ML. Sleep disturbances as an evidence-based suicide risk factor. Curr Psychiatry Rep 2015; 17(3): 554

6 Appleby L. The National Confidential Inquiry into Suicide and Homicide by People with Mental IIIness Annual Report 2015: England, Northern Ireland, Scotland and Wales. University of Manchester, 2015.

7 Khan M, Daw R, Hampson M. OP79. Do the Right Thing: How to Judge a Good Ward. Royal College of Psychiatrists, 2011.

8 National Institute of Health and Care Excellence. Safe Staffing for Nursing in Adult Inpatient Wards in Acute Hospitals. NICE, 2014.

9 Buysse DJ, Reynolds CF, Monk TH, Berman SR, Kupfer DJ. The Pittsburgh Sleep Quality Index: a new instrument for psychiatric practice and research. Psychiatry Res 1989; 28(2): 193-213.

10 Chung F, Subramanyam R, Liao P, Sasaki E, Shapiro C, Sun Y. High STOP-BANG score indicates a high probability of obstructive sleep apnoea. Br J Anaesth 2012; 108(5): 768-75.

11 Bradley AJ, Webb-Mitchell R, Hazu A, Slater N, Middleton B, Gallagher $P$, et al. Sleep and circadian rhythm disturbance in bipolar disorder. Psychol Med 2017; 47(9): 1678-89.

12 van Hees VT, Sabia S, Anderson KN, Denton SJ, Oliver J, Catt M, et al. A novel, open access method to assess sleep duration using a wristworn accelerometer. PLOS ONE 2015; 10(11): e0142533.

13 Cole JC, Motivala SJ, Buysse DJ, Oxman MN, Levin MJ, Irwin MR Validation of a 3-factor scoring model for the Pittsburgh sleep quality index in older adults. Sleep 2006; 29(1): 112-6.

14 Gupta MA, Simpson FC. Obstructive sleep apnea and psychiatric disorders: a systematic review. J Clin Sleep Med 2015; 11(2): 165-75.

15 Anderson KN, Waton T, Armstrong D, Watkinson HM, Mackin P. Sleep disordered breathing in community psychiatric patients. Eur J Psychiatry 2012; 26(2): 86-95

16 Foley DL, Morley KI. Systematic review of early cardiometabolic outcomes of the first treated episode of psychosis. Arch Gen Psychiatry 2011; 68(6): 609-16.

17 De Hert M, Correll CU, Bobes J, Cetkovich-Bakmas M, Cohen D, Asai I, et al. Physical illness in patients with severe mental disorders. I. Prevalence, impact of medications and disparities in health care. World Psychiatry 2011; 10(1): 52-77.

18 Berglund B, Lindvall T, Schwela DH. Guidelines for Community Noise. World Health Organization, 1999.

19 Busch-Vishniac IJ, West JE, Barnhill C, Hunter T, Orellana D, Chivukula R. Noise levels in Johns Hopkins Hospital. J Acoust Soc Am 2005; 118: 3629-45.

20 McClung CA. How might circadian rhythms control mood? Let me count the ways... Biol Psychiatry 2013; 74(4): 242-9.

21 Wulff K, Dijk DJ, Middleton B, Foster RG, Joyce EM. Sleep and circadian rhythm disruption in schizophrenia. Br J Psychiatry 2012; 200(4): 308-16.

22 Krahn LE, Lin SC, Wisbey J, Rummans TA, O'Connor MK. Assessing sleep in psychiatric inpatients: nurse and patient report versus wrist actigraphy. Ann Clin Psychiatry 1997; 9(4): 203-10. 